\title{
Ecosistemas de producción camaroneros: Estudios y proyecciones para la gestión de costos
}

\section{Shrimp production ecosystems: Studies and projections for cost management}

Rosana de Jesús Eras-Agila

Universidad Técnica de Machala, Machala, Ecuador

reras@utmachala.edu.ec

https://orcid.org/0000-0003-0172-7271

Rosana Meleán-Romero

Universidad del Zulia, Maracaibo, Venezuela

rosanamelean@gmail.com

melean_rosana@fces.luz.edu.ve

(D) https://orcid.org/0000-0001-8779-738X

Recepción: 22/07/2021 | Aceptación: 22/10/2021 | Publicación: 30/10/2021

Cómo citar (APA, séptima edición):

Eras-Agila, R. J., y Meleán-Romero, R. (2021). Ecosistemas de producción camaroneros: Estudios y proyecciones para la gestión de costos. INNOVA Research Journal, 6(3.1), 41-59.

https://doi.org/10.33890/innova.v6.n3.1.2021.1833

\begin{abstract}
Resumen
La situación actual de pandemia, ha obligado a que las empresas sumen esfuerzos para mantener activa la producción. La investigación, caracteriza los ecosistemas de producción camaroneros, como base para la identificación de costos centrales en las operaciones productivas de empresas de Ecuador, teniendo como referencia los años 2019-2020. Apoyados en una metodología de corte documental, cualitativa, con documentos procedentes de revistas científicas scopus, web of science, scielo, latindex, entre otros relativos al tema, se identificaron las características de las diferentes etapas de producción del camarón, desde la preparación de estanques, piscinas, siembra, transferencia, engorde, hasta la cosecha del camarón. Los principales hallazgos demuestran que los ecosistemas estudiados manejan etapas asociadas al manejo y cosecha, precisando la adaptación y acondicionamiento de los suelos, gestión del aprovisionamiento de insumos y materia prima, resaltando la obtención de la larva de camarón para su cría y engorde. También son importantes procesos como el control de salinidad, temperatura, oxigeno, alimentadores, así como las labores de cosecha manual y mecanizada. En todos ellos se consumen recursos monetarios que al
\end{abstract}


clasificarlos se convierten en costos. Se rastrean en cada fase recursos asociados con la finalidad de proyectar los costos asociados al ciclo productivo del crustáceo, para su determinación futura bajo metodologías de costeo por proceso. La comprensión plena de estos ecosistemas se convierte en base para la organización, determinación y cálculo del costo; herramienta útil, en el proceso decisorio y en el cálculo de la rentabilidad real y la permanencia en los mercados.

Palabras claves: ecosistemas de producción; costos; camarón.

\begin{abstract}
The current pandemic situation has forced companies to join forces to keep production active. The research characterizes the shrimp production ecosystems, as a basis for the identification of central costs in the productive operations of companies in Ecuador, taking as a reference the years 20192020. Supported by a qualitative documentary methodology, with documents from scientific journals scopus, web of science, scielo, latindex, among others related to the subject, the characteristics of the different stages of shrimp production were identified, from the preparation of ponds, pools, planting, transfer, fattening, until the shrimp harvest. The main findings show that the studied ecosystems handle stages associated with management and harvest, specifying the adaptation and conditioning of the soils, management of the supply of inputs and raw materials, highlighting the obtaining of shrimp larvae for their rearing and fattening. Also important are processes such as the control of salinity, temperature, oxygen, feeders, as well as manual and mechanized harvesting tasks. In all of them monetary resources are consumed that when classified they become costs. Associated resources are tracked in each phase in order to project the costs associated with the crustacean's productive cycle, for future determination under process costing methodologies. The full understanding of these ecosystems becomes the basis for the organization, determination and calculation of the cost; Useful tool in the decision-making process and in the calculation of real profitability and permanence in the markets.
\end{abstract}

Keywords: production ecosystems; costs; shrimp.

\title{
Introducción
}

La Organización del sector Pesquero y Acuícola del Istmo Latinoamericano y el Sistema de Integración Centroamericana (SICA), al realizar una perspectiva de cultivo en la producción camaronera, señala países latinoamericanos, entre ellos Ecuador, tendrán un incremento en la producción de camarón, pero no por la expansión de nuevos cultivos, sino más bien originada por las "prácticas de gestión" adoptadas (Argandona, 2021). El crecimiento de la actividad acuícola en América Latina y el Caribe ha sido constante, destacándose la subregión de Sur América que muestra un mayor crecimiento comparando con América Central y el Caribe, al concentrar alrededor del 86\% de la producción acuícola total de la Región, seguida de América Central (13\%) y finalmente el Caribe (1\%). En términos del valor de dicha producción, Sur América supera con más de 11 veces el valor de la producción de América Central y más de 300 veces el valor de mercado de El Caribe UNIDO (2020) (Comisión Económica para América Latina y el CaribeCEPAL, 2019).

En la región latinoamericana, los avances técnicos suscitados desde 1973, han propiciado la reproducción artificial del camarón, adoptando técnicas para la cría intensiva, obteniendo especies 
como la Litopenaeus vannamei en área continental a principios de la década de 1980. Desde aquel momento, el cultivo comercial de esta especie, mostró una tendencia de rápido crecimiento convirtiéndose Brasil, Ecuador, México, Venezuela, Honduras, Guatemala, Nicaragua, del Pacífico, Perú, Colombia, Costa Rica, Panamá, El Salvador, Estados Unidos de América, Cuba, República Dominicana, en los principales países productores de camarón de esta especie, extendiéndose las técnicas de cultivo a otros países asiáticos (FAO, 2020).

El cultivo del camarón en cautiverio, suministra la mayor parte del volumen al mercado mundial, sin embargo, también ha sido afectado por el brote de enfermedades y variaciones de precios asociado al elevado volumen de producción en periodos 2018 y 2019, propiciando niveles bajos de mercado, reduciéndose con ello, la participación del camarón en el comercio mundial (FAO, 2020); afectándose también los precios de estos productos acuícolas, en los mercados internacionales (Uzcátegui et al., 2016,p.165).

Es evidente como la demanda y consumo de estos productos a nivel mundial, abre puertas para fortalecer la economía de un país. Sin embargo, grandes cambios políticos, económicos, sociales, tecnológicos, demográficos, culturales, ambientales, suceden vertiginosamente, por lo que las empresas deben estar alertas a la realidad, siendo preciso e importante analizar estos factores manifiestos a su alrededor, pues inciden en la gestión y ejecución de estrategias requeridas en el desarrollo de este tipo de actividad productiva cuyo producto tiene destino para países del mundo.

De este modo, el crecimiento de la actividad acuícola en América Latina y el Caribe ha sido constante, destacándose la subregión de Sur América que muestra un mayor crecimiento comparando con América Central y el Caribe, al concentrar alrededor del $86 \%$ de la producción acuícola total de la Región, seguida de América Central (13\%) y finalmente el Caribe (1\%). En términos del valor de dicha producción, Sur América supera con más de 11 veces el valor de la producción de América Central y más de 300 veces el valor de mercado de El Caribe (UNIDO, 2020).

Las bondades en recursos naturales y condiciones climáticas que posee Ecuador y debido a la existencia de ecosistemas que propician el cultivo de camarón convierte a la zona costera de mayor productividad biológica, transformando a la acuicultura en una de las actividades primarias productivas más importante, encontrándose en un dinamismo constante dentro de la industria agro alimentaria, ocupación con mayor viabilidad económica para la producción de alimento, no obstante, ha tenido que enfrentar fuertes crisis afectando drásticamente la producción del camarón, disminuyendo los beneficios económicos esperados.

La industria camaronera ha mantenido uno de los sistemas de producción de alimentos de más rápido crecimiento en las últimas décadas, el camarón blanco, es la principal especie para el cultivo en las costas del pacifico ecuatoriana, siendo de la familia Litopenaeus y la especie Litopenaeus vannamei por ser considerada la más resistente a cambios medioambientales, (Balod, 2021, p.9) menciona, que pese a la pandemia de Covid-19 Ecuador exportó 688 mil toneladas de camarón, generando divisas para el estado ecuatoriano de USD 3.823.53 millones según cifras del Banco Central de Ecuador, representando el 23,53\% de exportaciones no petroleras y el 18,90\% de las exportaciones totales. En comparación con el año 2019 las exportaciones crecieron en el 2020 
en un $7 \%$, aunque se habría estimado sin pandemia alcanzar el 18\%. Esto en un derrumbe de precios del camarón. Actualmente los productores estarían realizando todos los esfuerzos para diversificar la oferta incluyendo de ser posible valor agregado.

Esta actividad se ha diversificado e intensificado mediante los avances tecnológicos, generando seguridad alimentaria a nivel interno y externo del país de consumo, proyectándose alcanzar los mayores niveles de eficiencia en la producción, se estima que en el Ecuador hay aproximadamente 210.000 hectáreas dedicadas al cultivo de camarón distribuidas, el $60 \%$ en Guayas, el 15\% en El Oro, el 9\% en Manabí y el 7\% en Santa Elena (Dávila-López et al., 2019, p. 450-476). Bajo este contexto, el objetivo de la investigación, es caracterizar los ecosistemas de producción camaronera en Ecuador, tomando como referencia los años 2019-2020. La idea es identificar los costos centrales relativos a las operaciones productivas de estas empresas. En función de ello, se plantea como pregunta que motiva la necesidad del estudio, responder a ¿Cuáles son las características de los ecosistemas en la producción de camarón en Ecuador durante el período 20192020, considerando la producción en cautiverio?

\section{Metodología}

La investigación es descriptiva, de corte documental, cualitativa, apoyada en documentos procedentes de revistas científicas y de otros estudios realizados relativos al tema. Esto es basamento principal para la comprensión de los procesos desarrollados y la determinación y gestión de costos. Las bases teóricas fueron sustentadas por la metodología como teoría del método, (Tancara, 1993). Se parte de una revisión teórica documental realizada de manera sistemática. Se estableció como estrategia de búsqueda la exploración en bases de datos reconocidas internacionalmente, de preferencia: Scopus, Redalyc, Dialnet y SciELO y otras de acceso abierto. Se asumieron criterios de inclusión y selección de documentos: idioma (español), artículos relacionados con la temática planteada: ecosistemas de producción, costos de producción, empresas camaroneras precisando documentos seminales base y otros de reciente publicación.

Como resultado de la primera búsqueda se obtuvo información genérica relativa con la variable central, luego en una segunda revisión se definieron criterios de selección de documentos, de manera que se pudiese filtrar información pertinente asociada con las categorías de análisis definidas: ecosistemas de producción camaroneros, sistemas de producción camaroneros, costos de producción, estos últimos analizados desde la esencia de los recursos consumidos en las fases esenciales de dichos sistemas de producción primarios.

La información teórica recopilada fue dispuesta en una matriz de análisis en la que se incluyó información de los trabajos, considerando: año de publicación, autor, título, idioma, resumen, elementos centrales desarrollados en la investigación, principales aportes. Con el énfasis de realizar una selección más pertinente a la temática a investigar y más actualizada. En esta selección se trabajó con más interés en los artículos de revisión y fueron desechados los que no trataran las variables de estudio, quedando 45 trabajos relacionados al tema. Se analizaron los trabajos a partir de la descomposición del todo en sus elementos centrales, precisando los indicadores descritos en cada categoría de análisis, para una comprensión plena del fenómeno estudiado, recurriendo al conocimiento científico para la explicación del objeto de conocimiento. 


\section{Desarrollo}

\section{Ecosistemas de producción camaronera en Ecuador: breve caracterización}

La acuicultura como sector estratégico en la economía ecuatoriana se enfoca en el cultivo de camarón en cautiverio. Este sector, se ha mantenido aproximadamente por 50 años como un producto tradicional en el Ecuador. La regulación legal de la actividad, se establece en el artículo 7 de la Ley Orgánica para el Desarrollo de la Acuicultura y Pesca reformada el 17 de abril del 2020, donde se señala el numeral 2. Actividad acuícola, "es la que tiene por objeto la reproducción, cría, cultivo, procesamiento, comercialización interna y externa e investigación de recursos hidrobiológicos acuícolas y sus actividades conexas". Concretándose que la principal especie que se cultiva es la Litopenaeus vannamei, considerada la más resistente a cambios medioambientales, su crecimiento y calidad productiva le ha permitido posicionarse como líder en exportaciones no petroleras, siendo esta la producción de camarón en cautiverio de la cual, de acuerdo a (Argandona, 2021), existen 187 empresas entre productores y exportadoras afiliadas a la Cámara Nacional de Acuicultura de las cuales 144 corresponden a empresas productoras de camarón.

En este contexto es importante asumir un manejo adecuado de los procesos productivos del sector, basándose en diferentes fases de desarrollo del crustáceo, siendo necesario gestionar el cultivo en cada una de sus etapas productivas a través de decisiones tomadas día a día por personal con alto conocimiento en el área (Gonzaga et al., 2016). Al situarse en un sitial de gran importancia, conlleva exigencias que demandan la utilización necesaria de recursos como mano de obra, materiales, tecnológicos, económicos-financieros, los cuales deben ser controlados de forma exhaustiva mediante un tratamiento adecuado de los costos de producción para lograr altos niveles de productividad y eficiencia en las empresas productoras de camarón (Jácome et al., 2019, la caracterización de los procesos de producción camaronero es el basamento para la identificación de costos de producción centrales en las operaciones productivas, estos procesos son considerados de importancia para la rentabilidad organizacional.

Machado y Campos (2021), al respecto de los ecosistemas, los definen, como "una unidad básica de naturaleza, compuesta por un conjunto de organismos y el ambiente no viviente, cada uno influenciando las propiedades del otro y ambos necesarios para el mantenimiento de la vida, tal como la tenemos sobre la tierra", menciona, la interactuación entre organismos, plantas, animales, bacterias, algas, protozoos y hongos entre otros. Es decir, la transformación para favorecer el cultivo agrícola como un agroecosistema, que, a más de los factores naturales, las acciones del hombre, estarán acorde con la cultura, creencias, costumbres, motivaciones y tecnologías que utiliza, por lo que el suelo garantizará la seguridad alimentaria a la medida que el ser humano haga buen uso de los recursos neutralizando conflictos (Burbano-Orjuela, 2016).

Los ecosistemas agrícolas o agroecosistemas, se utilizan en la agricultura, con componentes similares, interacciones y funciones semejantes, comprendiendo como agroecosistemas los policultivos, monocultivos y sistemas mixtos, incluyendo los sistemas agropecuarios, agroforestales, agrosilvopastoriles, la acuicultura y las praderas, los pastizales y las tierras en barbecho. 
Ante este antecedente, podrían existir problemas para definir el valor exacto sobre el manejo de recursos naturales, por la falta de conocimiento en los valores atribuibles al bienestar del ser humano, al respecto Rodríguez et al. (2016) ejemplifica dicho problema, en la comparación de los costos de explotación y de conservación de los recursos naturales, habiéndose desarrollado y aplicado técnicas para su valoración, que permiten asignar un valor a los servicios ecosistémicos sin un mercado, como la regulación del clima, y el agua, la protección contra los peligros naturales y la biodiversidad; que será necesario conocer para su aplicabilidad.

Estudios realizados por (López, 2021) sostienen que en América Latina, en los ecosistemas terrestres la reducción de biodiversidad significa una pérdida de la riqueza intrínseca y genética, los costos que la degradación de la tierra representa para la región, equivale a USD 60000 millones anuales, afectando directamente la capacidad productiva y la posibilidad de explotar servicios ambientales en las zonas rurales, además, que los agroecosistemas que más impactos negativos han recibido son los asociados a la producción para el mercado Internacional, (CEPAL,2019), debido a la deforestación, contaminación de los suelos y aguas, estos, asociados a la producción de banano, palma africana, caña de azúcar, y otros cultivos exportables, adjudicando a la intervención humana como responsable del daño causado a más de la mitad de los ecosistemas terrestres, poniendo en riesgo a su propio bienestar, como también al resto de especies terrestres por los efectos negativos, esta degradación al ecosistema consideran que podrían incrementar significativamente en los próximos 50 años (Machado y Campos, 2021), lo que es urgente en la actualidad implementar estrategias para evitar las consecuencias previstas.

A propósito, los sistemas acuícolas, se clasifican en tres métodos: extensivo, semi-intensivo e intensivo. En esta investigación se abordarán los procesos de producción desarrollados en granjas, donde el ambiente organizacional es importante y determinante en las actividades y crecimiento del crustáceo. De acuerdo con (Niño, 2021), los ambientes donde se desarrolla la actividad productiva, incluyen variables que son controladas por la empresa, como los sistemas de producción, costos, y recursos. Sobre esta base, Ríos y Gómez (2008) plantean que los procesos de producción son actividades encargadas de generar y transformar la materia prima a través de la intervención de la mano de obra, tecnología, maquinarias diseñadas para un determinado fin, como es el caso de la actividad camaronera (Beltrán, 2017). Un proceso de producción, refiere la secuencia ordenada de actividades repetitivas, que se realizan en la organización por una persona, grupo o departamento, con la capacidad de transformar las entradas, en salidas o resultados de los recursos utilizados (Meleán-Romero et al., 2008).

Por tanto, los procesos son la base operativa de las organizaciones y es imperante la necesidad de disponer resultados organizacionales a las exigencias y expectativas de los clientes, (Hernández-Nariño et al., 2014) condiciones de un diseño lógico, planificado y controlado de los factores de producción, información, tecnología, financiamiento, mano de obra, materiales, insumos, maquinarias, instalaciones (Pesantez et al., 2021). El aprovechamiento de los recursos necesarios para la producción de bienes o servicios, tratando de minimizar o eliminar residuos y desechos que puedan contribuir a elevar el costo de producción, por tanto la utilización racional de los recursos garantizará ganancias a la organización (Mejía et al., 2016).

Conseguir que los miembros de la organización se concentren en sus funciones adecuados, de manera que se puedan representar los procesos de la organización como base para lograr mejorar 
la eficacia, eficiencia y flexibilidad es de prioridad. Además, se deberían desplegar elementos desde la racionalidad que permitan potenciar el desarrollo y concretar una caracterización que conlleve a su comprensión plena para efectos de toma de decisiones bajo puntos críticos de control previamente definidos (Limachi et al., 2018). La comprensión del procedimiento de trabajo se constituirá en una base para la determinación de los costos de producción, por ello la importancia de su organización y disposición de manera que se concrete la esencia misma de ellos.

\section{Tabla 1}

Ecosistemas e importancia del sector camaronero

\begin{tabular}{|c|c|c|c|}
\hline Autores & Tema & Propósito del estudio & $\begin{array}{c}\text { Características del proceso } \\
\text { productivo }\end{array}$ \\
\hline $\begin{array}{c}\text { (Sector } \\
\text { camaronero, } \\
2021)\end{array}$ & $\begin{array}{l}\text { Panorama General de } \\
\text { la Acuicultura Y el } \\
\text { Sector Camaronero } \\
\text { en el Mundo. Proceso } \\
\text { de producción de } \\
\text { Camarón. }\end{array}$ & $\begin{array}{l}\text { Analizar el uso de los } \\
\text { elementos que intervienen } \\
\text { en el proceso de } \\
\text { producción de camarón. }\end{array}$ & $\begin{array}{l}\text { Eficiencia en el uso de insumos } \\
\text { reducen los costos, genera mayores } \\
\text { ganancias y posibilita competir con } \\
\text { mejores precios. }\end{array}$ \\
\hline $\begin{array}{l}\text { (Aguirre et al., } \\
\text { 2019) } \\
\text { (Triviño y Zhinin, } \\
\text { 2018) }\end{array}$ & $\begin{array}{l}\text { Métodos de } \\
\text { producción en el } \\
\text { cultivo intensivo de } \\
\text { camarón blanco } \\
\text { (litopenaeus } \\
\text { vannamei) en baja } \\
\text { salinidad, una opción } \\
\text { para familias } \\
\text { emprendedoras. }\end{array}$ & $\begin{array}{l}\text { Brindar una nueva } \\
\text { actividad económica para } \\
\text { lugares marginados y } \\
\text { alejados a las zonas } \\
\text { costeras. }\end{array}$ & $\begin{array}{l}\text { Suelos arcillosos, no aptos para la } \\
\text { agricultura. Construcción de las } \\
\text { piscinas. Maquinaria agrícola, } \\
\text { Tractores. Construcción de muros, } \\
\text { Excavadora. Canales de evacuación de } \\
\text { aguas, puerta de cosecha. Tubería al } \\
\text { exterior. Alimento balanceado } \\
\text { Tamaño de larva en pre-cría } \\
\text { Bomba de sólidos. Equipos aereadores } \\
\text { Densidad de siembra. Personal } \\
\text { capacitado. Oxígeno, temperatura, pH } \\
\text { y parámetros químicos en el } \\
\text { agua como calcio, magnesio y potasio. }\end{array}$ \\
\hline $\begin{array}{c}\text { (Beltrán, María, } \\
\text { 2017) }\end{array}$ & $\begin{array}{l}\text { Innovación En El } \\
\text { Sector Acuícola }\end{array}$ & $\begin{array}{l}\text { El propósito del estudio, es } \\
\text { describir la agenda de } \\
\text { innovación que una } \\
\text { empresa acuícola, planea } \\
\text { llevar a cabo en el corto, } \\
\text { mediano y largo plazo, con } \\
\text { el } \\
\text { objetivo de mantener su } \\
\text { competitividad en el } \\
\text { mercado } \\
\text { internacional. }\end{array}$ & $\begin{array}{l}\text { Utilización del dispositivo electrónico } \\
\text { para la alimentación automática del } \\
\text { camarón, por la emisión de sonidos } \\
\text { que el camarón emite cuando siente la } \\
\text { necesidad de alimentares. }\end{array}$ \\
\hline (López, 2015) & $\begin{array}{l}\text { Cambio climático y } \\
\text { actividades } \\
\text { agropecuarias en } \\
\text { América Latina }\end{array}$ & $\begin{array}{l}\text { Presentar una síntesis de la } \\
\text { evidencia de los efectos } \\
\text { potenciales del cambio } \\
\text { climático sobre el sector } \\
\text { agropecuario, con énfasis } \\
\text { en América Latina. }\end{array}$ & $\begin{array}{l}\text { El impacto del cambio climático en los } \\
\text { costos de producción agropecuaria } \\
\text { hasta el } 2100 \text {, a una tasa de descuento } \\
\text { de } 4 \% \text { de forma acumulada hacia } 2050 \text {, } \\
\text { las pérdidas serían de alrededor de } \\
1,6 \% \text { y } 1,9 \% \text { del PIB. }\end{array}$ \\
\hline
\end{tabular}

Fuente: Elaboración propia en base a los autores referenciados 
Es evidente la importancia de estos sistemas de producción camaroneros en Ecuador y en otros países del mundo. Los productos resultantes aportan en esencia a la alimentación proteica de la población y se constituyen en materia prima para productos alimenticios también básicos en la dieta de los seres humanos. El mantener estos sistemas de manera eficiente y sustentable, aportara a las economías de las naciones, no solo al disponer de alimentos, sino al ver incrementadas fuentes de empleo, exportaciones e incrementos en los indicadores macroeconómicos de los países, cuya producción es representativa a nivel de la región latinoamericana.

\section{Ecosistemas de producción en el sector camaronero}

La importancia de conocer la actividad económica radica en la necesidad de identificar cada proceso que se genera y se ejecuta en la producción de camarón, de esta manera se estará en conocimiento de comprender y hacer el respectivo seguimiento de las actividades y control de los recursos necesarios que se utilizan durante el proceso desde la preparación de piscina siembra, desarrollo y cosecha del camarón, con el fin de gestionar los costos y luego asignar según corresponda en cada fase de producción. Se reconoce que hay pocos estudios que se ocupen en la gestión de costos de los procesos productivos en el área camaronera, los que existen tiene relación con el costo directo de los insumos al producto, como también aquellos que refieren al análisis del producto exportado. No obstante, a pesar de sus valiosas contribuciones, es fundamental estudiar la gestión de costos en los diversos procesos para su conocimiento y con basamento, realizar cambios e innovación que contribuyan al sostenimiento, eficiencia y eficacia empresarial.

$\mathrm{Al}$ considerar, el proceso productivo como un conjunto de tareas y procedimientos que la empresa necesita efectuar para producir bienes y servicios con el fin de satisfacer las demandas del mercado y la economía local, las organizaciones deben ajustarse a ciertos requerimientos optimizando los elementos más importantes como los factores de producción, proceso productivo, el producto, tipos de procesos sean estos; órdenes de pedidos o por procesos a considerarse en las diversas etapas, siendo necesario la adquisición y registros de materia prima, materiales e insumos, producción, desarrollo del producto hasta su comercialización, garantizando la planificación y controles adecuados de los recursos utilizados (Romero, 2014).

\section{Tabla 2}

Ecosistemas de producción camaronero ecuatoriano

\begin{tabular}{clll}
\hline Autores & \multicolumn{1}{c}{ Tema } & Propósito del estudio & $\begin{array}{c}\text { Consideraciones a tomar en cuenta } \\
\text { en los procesos productivos }\end{array}$ \\
\hline $\begin{array}{c}\text { (Romero, } \\
\text { 2014) }\end{array}$ & $\begin{array}{l}\text { Neoliberalismo e } \\
\text { industria camaronera en } \\
\text { Ecuador. }\end{array}$ & $\begin{array}{l}\text { Análisis de los efectos del } \\
\text { desarrollo de la industria } \\
\text { camaronera en el } \\
\text { ecosistema manglar, los } \\
\text { conflictos socio- } \\
\text { ambientales. }\end{array}$ & $\begin{array}{l}\text { Generación deterioro ambiental, } \\
\text { desprotección de las costas y pérdida } \\
\text { de recursos naturales, desempleo, } \\
\text { empobrecimiento de poblaciones } \\
\text { locales y costos al Estado. }\end{array}$ \\
(Argandona, & Sector Camaronero: & $\begin{array}{l}\text { Analizar de manera breve } \\
\text { las razones del crecimiento }\end{array}$ & $\begin{array}{l}\text { El valor agregado en el camarón no } \\
\text { tiene que ver con la industrialización, } \\
\text { sino con aspectos como la cría de }\end{array}$ \\
\hline
\end{tabular}




\begin{tabular}{|c|c|c|c|}
\hline Autores & Tema & Propósito del estudio & $\begin{array}{c}\text { Consideraciones a tomar en cuenta } \\
\text { en los procesos productivos }\end{array}$ \\
\hline & $\begin{array}{l}\text { Evolución y } \\
\text { proyección a corto } \\
\text { plazo. }\end{array}$ & $\begin{array}{l}\text { del sector camaronero en el } \\
\text { Ecuador. }\end{array}$ & $\begin{array}{l}\text { larvas, su alimentación y el desarrollo } \\
\text { tecnológico desde las piscinas. }\end{array}$ \\
\hline $\begin{array}{l}\text { (Karolay } \\
\text { Mishell et al., } \\
\text { 2019) }\end{array}$ & $\begin{array}{l}\text { Análisis de } \\
\text { rentabilidad económica } \\
\text { del camarón } \\
\text { (Litopenaeus } \\
\text { vannamei) en el sitio } \\
\text { Balao Chico, provincia } \\
\text { del Guayas. }\end{array}$ & $\begin{array}{l}\text { Análisis financiero } \\
\text { mediante un flujo de caja y } \\
\text { la obtención de indicadores } \\
\text { que permita determinar la } \\
\text { rentabilidad del cultivo de } \\
\text { camarón el sitio Balao } \\
\text { Chico, en la provincia del } \\
\text { Guayas. }\end{array}$ & $\begin{array}{l}\text {-Inversiones en turbinas para motores. } \\
\text {-Motores estacionarios. } \\
\text {-Instalación Energía Eléctrica } \\
\text {-Aereadores } \\
\text { - Casetas para Aereadores }\end{array}$ \\
\hline $\begin{array}{l}\text { (Pesantez et } \\
\text { al., 2021) }\end{array}$ & $\begin{array}{l}\text { Integración de } \\
\text { Sistemas Solares } \\
\text { Fotovoltaicos en el } \\
\text { Sector Camaronero } \\
\text { Intensivo y Extensivo } \\
\text { del Ecuador: Caso de } \\
\text { Estudio en la Provincia } \\
\text { de El Oro. }\end{array}$ & $\begin{array}{l}\text { Considerar la energía } \\
\text { como un recurso } \\
\text { gestionable de forma } \\
\text { inteligente mediante la } \\
\text { introducción de acciones de } \\
\text { eficiencia energética }\end{array}$ & $\begin{array}{l}\text { Los resultados demuestran que la } \\
\text { integración de sistemas de generación } \\
\text { solar puede satisfacer parcialmente la } \\
\text { demanda eléctrica de los cultivos } \\
\text { intensivos y extensivos de camarones } \\
\text { de la provincia de El Oro y permitirá } \\
\text { reducir significativamente los costos } \\
\text { de operación e incrementar la } \\
\text { competitividad de los sectores } \\
\text { productivos }\end{array}$ \\
\hline $\begin{array}{c}\text { (Machado y } \\
\text { Campos, 2008) }\end{array}$ & $\begin{array}{l}\text { Reflexiones acerca de } \\
\text { los ecosistemas } \\
\text { agrícolas y la necesidad } \\
\text { de su conservación. }\end{array}$ & $\begin{array}{l}\text { Reflexionar sobre el uso } \\
\text { que se hace de los } \\
\text { ecosistemas para satisfacer } \\
\text { las necesidades humanas y } \\
\text { los impactos que ha estado } \\
\text { teniendo a largo plazo }\end{array}$ & $\begin{array}{l}\text { Para agroecosistemas estables, será } \\
\text { necesario diversificación de los } \\
\text { hábitats mediante rotaciones, los } \\
\text { policultivos, los cultivos de cobertura, } \\
\text { el mantenimiento de vegetación, } \\
\text { fertilización orgánica, laboreos } \\
\text { superficiales que incrementa la } \\
\text { biodiversidad. }\end{array}$ \\
\hline $\begin{array}{l}\text { (García et al., } \\
\text { 2016) }\end{array}$ & $\begin{array}{l}\text { La valoración de los } \\
\text { servicios ecosistémicos } \\
\text { en los ecosistemas } \\
\text { forestales: un caso de } \\
\text { estudio en Los Alpes } \\
\text { Italianos. }\end{array}$ & $\begin{array}{l}\text { Desarrollar un método que } \\
\text { proporcione información } \\
\text { detallada y precisa sobre la } \\
\text { distribución espacial de los } \\
\text { valores correspondientes a } \\
\text { los servicios ecosistémicos. }\end{array}$ & $\begin{array}{l}\text { Valorar los servicios ecosistémicos } \\
\text { como el uso del suelo, el tipo forestal } \\
\text { y la actividad turística de la zona. La } \\
\text { distribución espacial permite } \\
\text { conocimiento útil para los } \\
\text { responsables encargados de la toma } \\
\text { de decisiones. }\end{array}$ \\
\hline
\end{tabular}

Fuente: elaboración propia con base a los autores referenciados

\section{Etapas y actividades asociadas}

Toda actividad económica requiere de etapas y procesos, es así que la actividad camaronera mantiene diversas actividades en cada etapa de producción, mediante procesos que conllevan a lograr sus metas, este proceso sugiere varios impactos potenciales en el medioambiente (Rodríguez et al., 2016), lo que pueden suceder en dos fases secuenciales. 
Siendo el primero, la ubicación, diseño y construcción de las piscinas; el segundo durante su operación, constituyéndose el efecto más importante, referido a este sector, es el establecimiento de los cultivos en ecosistemas frágiles, mientras más extensiva es la explotación, requerirá mayor extensión de áreas cultivadas (Moreno, 2010). A continuación, se presenta las fases de la producción del camarón en cautiverio.

\section{Gráfico 1}

Fases de producción del camarón en cautiverio

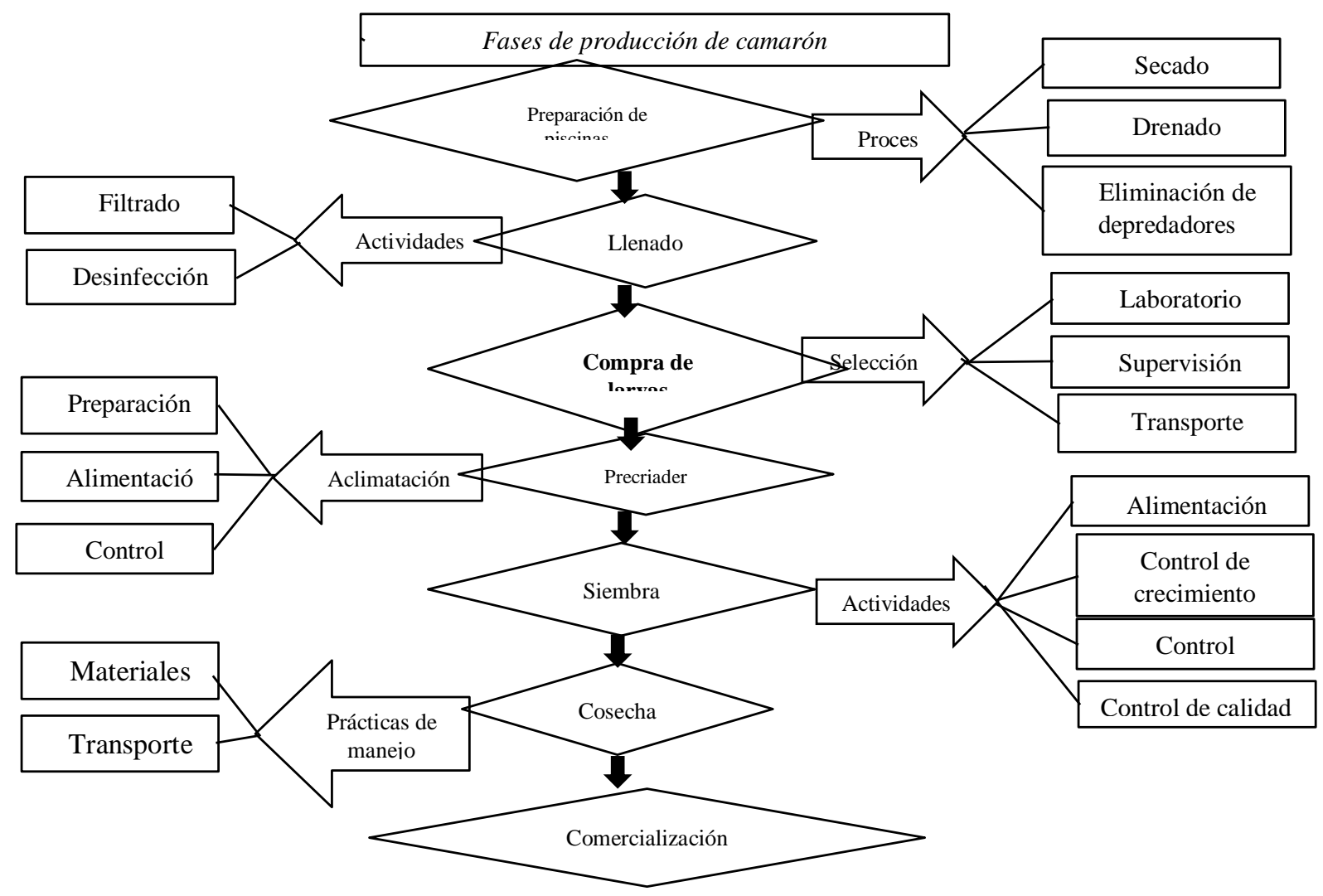

Fuente: (Cuéllar-Anjel, 2010)

En el proceso productivo de camarón en cautiverio consiste de diversas fases de acuerdo a (Villamar,2004); (Godínez-Siordia, et al., 2011); (Hernández y García, 2015) entre las más relevantes están, la construcción y preparación de piscinas o estaques, adquisición y siembra de semilla (post-larva, aclimatación, proceso de engorde, control de enfermedades, alimentación, mano de obra calificada; con estándares apropiados y su funcionamiento eficiente, se construirán adicionalmente canal de drenajes que servirán para recoger las aguas de las cosechas y recircular hacia el interior que servirá para minimizar los posibles impactos al ecosistema. De acuerdo a (Cuéllar-Anjel, 2010), refieren a diversos procesos como el drenado, secado, manejo de sedimentos, la desinfección del estanque comprende limpieza, evaluación del fondo, son actividades que contribuyen a disminuir los riesgos de enfermedades, procurando no dañar la flora y fauna silvestre 
al incrementar la aireación del suelo estimulando la descomposición de la materia orgánica. La limpieza del estaque se realiza después de la cosecha, combinando con la acción de radiación solar. De acuerdo con Boyd et al. (2005) y (Cuéllar-Anjel, 2010), los tablones deberán ser cambiados de ser necesarios. En el llenado del estanque, el agua debe cumplir estándares de calidad, rica en nutrientes orgánicos (Aguirre et al., 2019) se utilizan filtros y mallas para contener animales depredadores en el agua que ingresa al estanque, la supervisión estricta del técnico, garantiza la limpieza. Para la desinfección se utiliza instrumentos, materiales de operación, equipos, vehículos y otros elementos (Miranda y Toirac, 2010). Para la compra de larvas, de acuerdo a (Cuéllar-Anjel, 2010) consiste en la selección de laboratorios, supervisión y transporte. Siendo que la elección de laboratorio, es primordial, deben contar con las respectivas autorizaciones de funcionamiento para que las postlarvas sean adquiridas y certifiquen esa resistencia y/o ausencia de enfermedades. La supervisión, corresponde al personal técnico, verifica y controla in situ la sobrevivencia de las postlarvas sobre alimento, oxígeno, salinidad, certificando la buena salud y calidad del crustáceo, considerables para el éxito del cultivo.

La fase de Precriadero, según Boyd et al. (2005), esta fase consiste en preparación de estanques, alimentación y control fitosanitario, la calidad de agua, salinidad, temperatura, para poder ubicar las postlarvas. La alimentación ayudará a la postlarvas a tener más energía para soportar el estrés ocasionado por la aclimatación, el alimento se lo calcula en base al peso promedio del crustáceo (Faillace et al., 2016). El control fitosanitario, requiere del conocimiento de diversos aspectos biológicos de las especies en producción, será pertinente tomar medidas apropiadas y hacer ajustes oportunos, debido a la variabilidad ambiental y falta de control (Jory, 2020). De ahí que para mantenerse en equilibrio necesita subsidios como lo son fertilizantes, cal, los recambios de agua y el alimento balanceado. En la siembra, se considera densidades que no superen la capacidad de carga del estanque como también implantando técnicas de manipulación de post-larvas, evitando estrés, y pérdidas económicas, como la afectación ambiental, la utilización de aireadores en estanques es necesaria para el desarrollo del camarón. (Dávila-López et al., 2019), la densidad de la siembra es primordial dependiendo del sistema de cultivo adoptado.

En la etapa de cría y engorde de camarones, de acuerdo a Triviño y Zhinin (2021) se realiza con un protocolo de trabajo, al ser una especie que vive en agua salada y dulce, su mayor producción en el Ecuador es en estanques y agua salada, luego de la etapa de aclimatación le permite tener una buena salud al crustáceo, permitiendo realizar cultivos de 3 a 4 veces en el año, (Aguirre et al., 2019) los procesos son una serie de combinaciones de recursos de manera sistemática. identificando etapas o fases en las que requieren el uso de recursos como mano de obra, materiales, insumos, tecnología, infraestructura; que son requeridas para la transformación en el crecimiento e incremento del valor del ser vivo como es el camarón (Meleán y Ferrer, 2019) (Meleán y Ferrer, 2019).

El alimento según Sorroza et al. (2018), será acorde al crecimiento del crustáceo por cuanto el alimento concentrado representa entre el $50 \%$ y $70 \%$ del costo de producción, este insumo será lo más eficiente en términos nutricionales, por lo tanto, su administración debe ser óptima (Faillace et al., 2016). El personal deberá ser constante en las granjas. Se capacitará al personal que laborará en los procesos de cría en las piscinas de producción, sobre conceptos básicos mínimos de contabilidad de costos, para el manejo de bodega, inventarios, relaciones humanas, roles de pago, elaboración de informes, manejo de personal, nutrición animal, principios éticos, responsabilidad 
compartida, (Ballesteros et al., 2015). El control fitosanitario y el de calidad, de acuerdo a (Boyd et al., 2005) y (Cuéllar-Anjel et al., 2010) el sitio seleccionado es el primer paso importante que se da, asegurando una producción exitosa, previniendo riesgos de peligro para los alimentos de consumo humano. La desinfección constante de instalaciones, equipo y utensilios es una medida que se debería aplicar en todas las granjas, Es importante que, al utilizar alimento artificial, sea proveniente de un establecimiento certificado que cumpla con la implementación de un programa de aseguramiento de control de calidad e inocuidad como por ejemplo: Manual de buenas prácticas de manejo (BPM), Análisis de peligros y puntos de control, (APPCC/HACCP = Hazard Analysis Critical Control Point (por sus siglas en inglés).

Para la cosecha, de acuerdo a (Cuéllar-Anjel et al., 2010) en esta fase, las Buenas Prácticas de Manejo (BPM), en la granja, se garantiza la inocuidad del producto cosechado. El mantenimiento de registros adecuados es necesario, por cada recipiente de cosecha, con respecto a la cantidad de hielo, cantidad de camarón, tiempo de captura, tiempo de llenado de recipiente, el camarón debe ser cubierto de hielo inmediatamente después de haber sido cosechado, con lo que inicia la cadena de frio; capas alternas de hielo y camarón son recomendadas para evitar bolsas de altas temperatura o fluctuaciones en la temperatura. En la comercialización, de acuerdo a (Villamar, 2004) se transportan en gavetas colocando capas de hielo triturado colocando sellos de seguridad numerados en la puerta del camión, en el transporte viajarán con escolta de seguridad. La comercialización se realizará exclusivamente en los mercados más rentables.

\section{Procesos de trabajo en la industria camaronera: base para la determinación de costos y toma de decisiones}

La importancia de considerar la gestión de costos en la economía de una organización depende del papel relevante de los directivos y del desarrollo productivo, entendiéndose que del desempeño gerencial, se orientará el crecimiento o estancamiento de la empresa, por esta razón es de gran relevancia conocer las formas de producción y la capacidad instalada; estructura de costo en cuanto a sistemas de acumulación y elementos que integran el costo del producto; técnicas, estrategias y métodos utilizados para la distribución de los costos generales; planificación y controles implementados para garantizar el buen manejo de los recursos, manteniendo las especificaciones de calidad. Establecer relación entre los sistemas de producción que se aplica, costos y estrategias, gerenciados adecuadamente mediante formas de trabajo, creatividad, ingenio para formular estrategias permitirán optimizar los recursos, encaminados a mejorar los costos que logren productividad y competitividad en el mercado, (Meleán y Ferrer, 2019). El éxito de toda organización depende, cada vez más, del desarrollo eficiente de los procesos empresariales, alineados con su estrategia, misión y objetivos. En este sentido, autores como (Rincón-Soto et al., 2019) refieren los componentes del costo, como materiales, mano de obra y los costos comunes de explotación. Estos elementos son importantes de conocer en el sector camaronero, particularmente en las actividades que este desarrolla.

\section{Materiales e insumos requeridos en los procesos de transformación}

Con respecto a la materia prima que se transforma en el proceso de producción del camarón, esta incorpora todos los principales recursos, elementos físicos imprescindibles para el consumo en el proceso productivo, estos se transforman en productos terminados. Materia prima directa; 
conforma la parte integral del bien en producción y es plenamente identificable y determinable en el producto terminado (Arellano et al., 2017), desde este sistema se determina la compra de materiales y la salida del producto terminado. En el caso de la producción de camarón, la materia prima como es la postlarva, proviene de laboratorios para ser ubicados en reservorio para su aclimatación y posteriormente trasladarse a las piscinas o estanques de engorde donde se proveerá de insumos hasta la cosecha.

\section{Fuerza de trabajo en el proceso de producción}

La mano de obra, representa el esfuerzo físico o mental empleado en la transformación de la materia prima en producto final, la administración determinará la relación de fuerza laboral en las diversas áreas de la entidad, en los procesos de transformación de un producto nuevo (Orozco et al., 2004). Los sectores económicos (Varoni y Rodríguez, 2017) tienen su basamento legal en el Código de trabajo que regula las relaciones empleador - trabajador, cada año se emite una tabla sectorial donde constan las actividades por sectores económicos especificando área económica, actividad que realiza cada trabajador, descripción del cargo, código que corresponde según la función que desempeña para su ingreso al Instituto Ecuatoriano de Seguridad Social, y el salario mínimo establecido al que tendrá derecho el trabajador, se estima que la actividad camaronera emplea una población económicamente activa, aproximadamente de 200.000 empleados de manera directa (Cruz y Meza, 2021).

\section{Tabla 3}

Capital de trabajo de producción en producción de camarón ecuatoriano

\begin{tabular}{|c|c|c|c|}
\hline Autores & Tema & Propósito del estudio & $\begin{array}{l}\text { Consideraciones relevantes de } \\
\text { estudios previos }\end{array}$ \\
\hline $\begin{array}{l}\text { (Rincón-Soto et } \\
\text { al., 2019) }\end{array}$ & $\begin{array}{l}\text { Clasificación teórica de } \\
\text { los costos }\end{array}$ & $\begin{array}{l}\text { Explicar teóricamente } \\
\text { las diferentes estructuras } \\
\text { de categorización de } \\
\text { costos. }\end{array}$ & $\begin{array}{l}\text { Una de las estrategias de la } \\
\text { contabilidad, volver visible un atributo, } \\
\text { es revelarlo, explicarlo, desglosarlo, } \\
\text { darle significado y categorizarlo. }\end{array}$ \\
\hline $\begin{array}{l}\text { (Dávila-López } \\
\text { et al., 2019) } \\
\text { (Hernández y } \\
\text { García, 2015) } \\
\text { (Godínez- } \\
\text { Siordia, et al., } \\
\text { 2011) } \\
\text { (Cuellar-Anjel, } \\
\text { 2010) }\end{array}$ & $\begin{array}{l}\text { Análisis de rentabilidad } \\
\text { económica del camarón } \\
\text { (Litopenaeus } \\
\text { vannamei) en el sitio } \\
\text { Balao Chico, provincia } \\
\text { del Guayas. }\end{array}$ & $\begin{array}{l}\text { Análisis financiero } \\
\text { mediante un flujo de caja } \\
\text { y la obtención de } \\
\text { indicadores que permita } \\
\text { determinar la } \\
\text { rentabilidad del cultivo } \\
\text { de camarón el sitio Balao } \\
\text { Chico, en la provincia } \\
\text { del Guayas }\end{array}$ & $\begin{array}{l}\text { Materia Prima e Insumos } \\
\text { Nómina Producción y Administración } \\
\text { Costos Indirectos de Producción } \\
\text { Gastos Administrativos } \\
\text { Larva } \\
\text { Alimento de balanceado } \\
\text { Fertilizantes }\end{array}$ \\
\hline $\begin{array}{l}\text { (Mejía et } \\
\text { al.,2016) }\end{array}$ & $\begin{array}{l}\text { Análisis de las } \\
\text { variables críticas de un } \\
\text { sistema productivo a } \\
\text { través de la Dinámica } \\
\text { de Sistemas. }\end{array}$ & $\begin{array}{l}\text { Analizar diferentes } \\
\text { propuestas de decisión } \\
\text { para su mejoramiento. }\end{array}$ & $\begin{array}{l}\text { Ventajas del sistema Productivo: } \\
\text {-Reducción de inventario notable } \\
\text { reducción de costos. } \\
\text {-Información brindada por la persona } \\
\text { más preparada en el tema. } \\
\text { - Pronóstico visionario. } \\
\text { - No existe secreto y se fomenta la } \\
\text { comunicación. }\end{array}$ \\
\hline
\end{tabular}

Fuente: Elaboración propia en base a los autores referenciados 


\section{Otros recursos indirectos}

La materia prima indirecta forma parte de la elaboración de un producto, su relevancia frente a los directos es que no se puede identificar y determinar con exactitud en la producción del bien, siendo parte de los costos indirectos de producción (Arellano et al.,2017), mientras que la mano de obra Indirecta: está constituida por un costo significativo en la producción de un bien, por horas extras, beneficios sociales, sueldos, supervisores, técnicos de producción. (Meleán y Ferrer, 2019), ambos forman parte del costo de producción y se suman como costos indirectos de fabricación. En la producción de camarón, los costos indirectos incluyen la depreciación de la tecnología adoptada, aunque presenta condiciones favorables al medio ambiente, también disminuye los costos, contribuye a reducir los riesgos de contaminación, de tal manera que los gobiernos como la industria camaronera encaminan esfuerzos para implementar la tecnología en el proceso productivo como prevención a la salinización del subsuelo, medio ambiente y productividad. Al mantener buenas prácticas, prevalece la mayor bioseguridad e incrementa la eficacia en reducción de costos. La capacidad del sector productivo para generar o implementar nuevas tecnologías, como establecer normas innovadoras relacionadas con el conocimiento, gestión e innovación, a través de una larga trayectoria tecnológica que en base a las necesidades se iría incorporando en la industria, obteniendo mejoras mediante atributos técnicos, y económicos (Perozo y Nava, 2005).

\section{Tabla 4}

Proceso productivo de camarón

\begin{tabular}{|c|c|c|c|}
\hline Fases & Materiales e insumos & Mano de obra & $\begin{array}{l}\text { Otros recursos } \\
\text { indirectos }\end{array}$ \\
\hline $\begin{array}{l}\text { Preparación } \\
\text { de piscinas }\end{array}$ & $\begin{array}{l}\text { Fertilizantes, cal, mallas, tablones, } \\
\text { desinfectantes. }\end{array}$ & $\begin{array}{l}\text { Aplicación de insumos, } \\
\text { limpieza de las piscinas, } \\
\text { mantenimiento de } \\
\text { compuertas y ubicación de } \\
\text { mallas y tablones. }\end{array}$ & $\begin{array}{l}\text { Arriendos, combustibles, } \\
\text { lubricantes, depreciación, } \\
\text { sistemas de seguridad }\end{array}$ \\
\hline $\begin{array}{l}\text { Adquisición } \\
\text { de larva }\end{array}$ & $\begin{array}{l}\text { Semillas post-larva de camarón, } \\
\text { recurso económico. }\end{array}$ & $\begin{array}{l}\text { Transporte y supervisión } \\
\text { del estado de salud. }\end{array}$ & Seguridad. \\
\hline Pre-criaderos & $\begin{array}{l}\text { Estanques, agua, post-larvas de } \\
\text { camarón, vitaminas, medicamentos, } \\
\text { balanceados }\end{array}$ & $\begin{array}{l}\text { Alimentación, monitoreo y } \\
\text { supervisión de salinidad, } \\
\text { temperatura y estado de } \\
\text { salud }\end{array}$ & $\begin{array}{l}\text { Depreciaciones y } \\
\text { servicios básicos, medios } \\
\text { de comunicación } \\
\text { lubricantes, combustible, } \\
\text { seguridad. }\end{array}$ \\
\hline $\begin{array}{l}\text { Siembra pre- } \\
\text { juveniles }\end{array}$ & $\begin{array}{l}\text { Agua, aireadores, balanceado, } \\
\text { vitaminas, medicamentos, bombas, } \\
\text { motores, balanzas, laboratorio, } \\
\text { alimentadoras, botes, comederos. }\end{array}$ & $\begin{array}{l}\text { Alimentación, monitoreo } \\
\text { de temperatura, y } \\
\text { supervisión del estado de } \\
\text { salud y desarrollo, } \\
\text { aplicación de insumos. }\end{array}$ & $\begin{array}{l}\text { Depreciaciones y } \\
\text { servicios básicos } \\
\text { lubricantes y combustible, } \\
\text { seguridad. }\end{array}$ \\
\hline
\end{tabular}




\begin{tabular}{|c|c|c|c|}
\hline Fases & Materiales e insumos & Mano de obra & $\begin{array}{l}\text { Otros recursos } \\
\text { indirectos }\end{array}$ \\
\hline $\begin{array}{l}\text { Cosecha de } \\
\text { camarón }\end{array}$ & $\begin{array}{l}\text { Tubería, acoples, cosechadoras, tinas, } \\
\text { gavetas caladas, balanza, termómetro, } \\
\text { hielo, oxigenó metro, atarraya, bomba, } \\
\text { generador eléctrico instalación } \\
\text { eléctrica, focos reflectores, tablero y } \\
\text { hojas de muestreo. }\end{array}$ & $\begin{array}{l}\text { Muestreos y análisis } \\
\text { (previo a la cosecha), } \\
\text { personal de pesca, limpieza } \\
\text { de compuertas, transporte. }\end{array}$ & $\begin{array}{l}\text { Depreciaciones, } \\
\text { lubricantes combustible, } \\
\text { servicios básicos, } \\
\text { seguridad }\end{array}$ \\
\hline
\end{tabular}

Fuente: Elaboración propia

Los procesos productivos de acuerdo a (Hernández-Nariño et al., 2014), requieren de diversos materiales, e insumos conformando los costos de producción en relación a materiales: balanza de pesajes, tinas, tanques, recipientes, mallas, comederos, bandeja metálica; alimentadoras al abordar la tecnología en la producción.

Los insumos utilizados en la actividad camaronera son: alimentos balanceados, fertilizantes, cal, melaza; en relación a infraestructura: almacenamiento de balanceado, estación de bombeo, almacenamiento de combustible; para el recambio de agua se colocan mallas alrededor de las compuertas impidiendo que el camarón salga mientras las compuertas están abiertas cuando se desagua. Por la alta productividad y producción de materia orgánica que se lograría mantener con el cuidado de los ecosistemas en vista de que contribuye al sostenimiento de una red alimentaria de los cuerpos de agua en sus alrededores (Mendoza et al., 2019), es de gran importancia para las empresas productoras del sector acuícola, mantener una adecuada gestión de costos en los procesos productivos.

La articulación de estos elementos centrales en el análisis de los costos de producción, permite a los gerentes disponer de información pertinente, oportuna y veraz relativa a la materia prima e insumos, mano de obra y otros recursos que se inserten en la producción camaronera. Esta información clasificada de manera precisa arrojará datos reales sobre la rentabilidad y eficiencia productiva y económica de estos ecosistemas de producción tan importantes para el país, y por ende para el mundo.

\section{Conclusiones}

La identificación de las operaciones propias de los ecosistemas de producción del sector camaronero, permiten fundamentar las diversas actividades contenidas en las fases o etapas de los procesos desarrollados. Estas actividades permiten rastrear e identificar y precisar en cada fase del proceso los recursos requeridos con el fin de gestionar los costos en cada fase de producción, respetando metodologías de gestión por procesos y actividades.

Las revisiones teóricas realizadas en este trabajo, enfatizan la importancia de reconocer el comportamiento de estos ecosistemas de producción acuícolas; la adecuada utilización de los recursos en las actividades productivas es fundamental para generar rendimientos eficientes y propiciar la toma de decisiones centrados en datos organizados y sistematizados, de manera que las organizaciones puedan ser sustentables y sostenibles a nivel de su desarrollo productivo. 
Las diversas fases de producción que se requieren para la cría y engorde de camarón en cautiverio con fines de comercialización generan una gran responsabilidad por parte de las empresas para proteger el ecosistema mediante una gestión de costos adecuada, que redunde en el buen uso de los recursos en las operaciones productivas. Esta gestión de costos, requiere la identificación, organización, clasificación y asignación de los recursos monetarios consumidos a las actividades y procesos, de manera que se pueda disponer de información relativa a la materia prima, mano de obra y otros recursos consumidos, sean estos directamente asociados con la producción o bajo una relación indirecta. En todo caso, el reconocer estos ecosistemas de producción, la información relativa a costos podrá organizarse y clasificarse de mejor manera, a la vez que permitirá identificar metodologías de trabajos existentes, que por puras o híbridas respondan a las necesidades propias de estos sistemas de producción.

La implementación de buenas prácticas de cultivo de camarón, el control, asignación y buen uso de los recursos por el aspecto ambiental y económico, la conservación de la biodiversidad biológica, la protección del ecosistema, impulsará a los productores a sumar esfuerzos para contar con un amplio programa de formación, encaminado al talento humano que participa durante el proceso, posibilitando el intercambio académico interinstitucional en el ámbito investigativo y formativo.

Los estudios previos de los procesos productivos en el sector camaronero, se convierten en base principal en el proceso de producción porque a través del conocimiento se gestiona y se genera la sostenibilidad de la industria camaronea, siendo vital conocer el comportamiento de costo de la empresa, para analizar sus procesos clave y todas las actividades, medir y vincular las actividades con los productos y servicios que son accesorios determinantes para establecer el costo del producto y la entidad pueda optar por decisiones acertadas y oportunas.

\section{Referencias bibliográficas}

Aguirre, D., Maridueña, M., Ching, C., y Pérez, O. (2019). Métodos de producción en el cultivo intensivo de camarón blanco (litopenaeus vannamei) en baja salinidad, una opción para familias emprendedoras. Ciencia y Tecnología, Revista Científica Ciencia Y Tecnología, 19(23). https://doi.org/10.47189/rcct.v19i23.256

Arellano Cepeda, Otto, Quispe Fernández, Gabith, Ayaviri Nina, Dante, \& Escobar Mamani, Fortunato. (2017). Estudio de la Aplicación del Método de Costos ABC en las Mypes del Ecuador. Revista de Investigaciones Altoandinas, 19(1), 33-46. https://bit.ly/3pNsfWh

Argandona, L. (2021). Sector Camaronero: Evolución y proyección a corto plazo. https://docplayer.es/54113635-Sector-camaronero-evolucion-y-proyeccion-a-cortoplazo.html

Ballesteros Silva, P. P., Castro, M. R., y Barrios Mendoza, H. M. (2015). Modelo de capacitación sobre logística integral de almacenamiento para autoservicios de retail. Scientia Et Technica, 20(1), 11. https://www.redalyc.org/pdf/849/84938609004.pdf

Balod, S. (2021). Acuacultura. Obtenido de Producción de camarón: https://issuu.com/revistacna/docs/edicion 139 
Beltrán, M. C. (2017). Innovación En El Sector Acuícola. Ra Ximhai, 15. https://www.redalyc.org/pdf/461/46154070020.pdf

Boyd, C., Kwei, C., Pantoja, C., Brock, J., Johnson, K., y Treece, G. (2005). Prácticas de Desarrollo Sostenible en Ambientes Costeros de Prioridad de los Ecosistemas del Golfo de California Marinas Recreativas y Maricultura. México: Graphic Services, Universidad de HawaiiHilo. https://www.crc.uri.edu/download/PKD_good_mgt_field_manual.pdf

Burbano-Orjuela, H. (2016). El suelo y su relación con los servicios ecosistémicos y la seguridad alimentaria. Revista de Ciencias Agrícolas, $\quad 33(2), \quad 8$. https://doi.org/10.22267/rcia.163302.58

CEPAL; Organización de las Nacionales Unidas para la Alimentación y la Agricultura. (2019). Perspectivas de la agricultura y del desarrollo rural en las Américas: una mirada hacia América Latina y el Caribe 2019-2020. San José, Costa Rica: Gráfica Litho-offset S.A. https://repositorio.cepal.org/bitstream/handle/11362/45111/1/CEPAL-FAO20192020_es.pdf

Cruz, A. F., y Meza, L. J. (12 de octubre de 2021). Caracterización de los factores de producción y productividad del sector camaronero en Ecuador. Obtenido de Producción de camarón: http://repositorio.uees.edu.ec/bitstream/123456789/2274/1/paper.pdf

Cuéllar-Anjel, J. C. (2010). Manual de buenas prácticas de manejo para el cultivo del camarón blanco Penaeus vannamei. Panamá: New Concept Publications, Inc. http://hdl.handle.net/1834/32462

Dávila-López, K., Carvajal-Romero, H., y Vite-Cevallos, H. (2019). Análisis de rentabilidad económica del camarón (Litopenaeus vannamei) en el sitio Balao Chico, provincia del Guayas. Polo del conocimiento, 5(01), 27. https://doi.org/10.23857/pc.v5i01.1233

Faillace, J., Vergara, R., y Suarez, A. (2016). Evaluación de una fórmula alimenticia para camarón de cultivo, (L. vannamei) con inclusión de proteína vegetal a base de harina de soya. AquaTIC, 18. https://www.redalyc.org/journal/494/49449812002/html/

Godínez-Siordia, D. E., Chávez-Sánchez, M. C., y Gómez-Jiménez, S. (2011). Acuicultura Epicontinental del Camarón Blanco del Pacífico, Litopenaeus (Boone, 1931). Tropical and Subtropical Agroecosystems, 9. https://www.redalyc.org/pdf/939/93915703004.pdf

Gonzaga, S., Morán, G., y Brito, B. (2016). Análisis exploratorio de buenas prácticas de manufactura del sector camaronero. Revista Científica de la Universidad de Cienfuegos, 8. http://scielo.sld.cu/scielo.php?script=sci_arttext\&pid=S2218-36202017000100004

Hernández Nariño, A., Medina León, A., Nogueira Rivera, D., Negrín-Sosa, A., y Marqués-León, M. (2014). La caracterización y clasificación de sistemas, un paso necesario en la gestión y mejora de procesos. Particularidades en organizaciones hospitalarias. Dyna. Retrieved from Procesos productivos: https://www.redalyc.org/pdf/496/49630405027.pdf

Hernández, E., y García, C. (2015). Desempeño ambiental de la camaronicultura en la región Caribe de Colombia desde una perspectiva de Análisis del Ciclo de Vida. Gestión y Ambiente, 23. https://www.redalyc.org/pdf/1694/169443282002.pdf

Hernández-Nariño, A., Medina-León, A., Nogueira-Rivera, D., Negrín-Sosa, E., y Marqués-León, M. (2014). La caracterización y clasificación de sistemas, un paso necesario en la gestión y mejora de procesos. Particularidades en organizaciones hospitalarias. Dyna, 81(184), 9. https://www.redalyc.org/pdf/496/49630405027.pdf 
Jácome, J., Quezada, C., Sánchez, O., Pérez, J., y Nirchio, M. (2019). Tilapia en Ecuador: paradoja entre la producción acuícola y la protección de la biodiversidad ecuatoriana. Peruana de Biología, 26(4), 8. http://doi.org/10.15381/rpb.v26i4.16343

Jory, D. (30 de noviembre de 2020). Manejo Integral del Alimento de Camarón, de Estanques de Producción Camaroneros, y Principios de Bioseguridad. Obtenido de Alimentación del camarón: https://interconsorcio.com/wp-content/uploads/2019/06/Manejo-integral-delalimento-de-camaron.pdf

Limachi, M., Naoki, K., y Armengot, L. (2018). Efecto de diferentes sistemas de producción de cacao de 3-4 años sobre la composición de un ensamble de hormigas terrestres. Ecología en Bolivia, 53(2), 15. http://www.scielo.org.bo/scielo.php?script=sci_arttext\&pid=S1605$25282018000200004 \& \operatorname{lng}=$ pt\&nrm $=$ iso

López, A. (11 de Julio de 2021). Cambio climático y actividades agropecuarias en América Latina. Obtenido de ecosistemas de la producción: https://repositorio.cepal.org/bitstream/handle/11362/39824/1/S1501286_es.pdf

Machado, H., y Campos, M. (11 de Julio de 2021). Reflexiones acerca de los ecosistemas agrícolas y la necesidad de su conservación. Obtenido de Ecosistemas de producción: http://scielo.sld.cu/scielo.php?script=sci_arttext\&pid=S0864-03942008000400005

Mejía, L., Arroyave, O., y González, R. (2016). Análisis de las variables críticas de un sistema productivo a través de la Dinámica de Sistemas. Scientia et Technica, 21(2), 10. https://doi.org/10.22517/23447214.9171

Meleán Romero, R., y Ferrer, M. A. (2019). Gestión de costos de producción en ganadería bovina del Municipio Valmore Rodríguez, Zulia-Venezuela. Revista De Ciencias Sociales, 25(4), 250-264. https://produccioncientificaluz.org/index.php/rcs/article/view/30531

Meleán-Romero, R, Bonomie-Sánchez, M.E, \& Rodríguez-Medina, G. (2008). Procesos productivos de la industria avícola zuliana: Fases de alimento, engorde y beneficio. Revista de la Facultad de Agronomía, 25(1), 160-184. https://bit.ly/3EBak9z

Mendoza Avilés, Henry Emilio, Betancourt Vera, Angélica Selena, \& Murillo Erazo, Franchesca Yuliana. (2019). Factores productivos que inciden en el desarrollo económico y comercial de la "Asociación de cangrejeros 6 de julio" en el Golfo de Guayaquil. Revista Universidad y Sociedad, 11(2), 387-394. https://bit.ly/3bnpaDQ

Miranda, J., y Toirac, L. (2010). Indicadores de Productividad para la Industria Dominicana. Ciencia y Sociedad. XXXV(2), 57. https://www.redalyc.org/pdf/870/87014563005.pdf

Moreno, F. (2010). Industria del camarón: su responsabilidad en la desaparición de los manglares y la contaminación acuática. REDVET. Revista Electrónica de Veterinaria, 11(5), 21. https://www.redalyc.org/pdf/636/63613160006.pdf

Niño, K. (11 de Octubre de 2021). Análisis para la gestión del riesgo de inundaciones en Bogotá: Un enfoque desde la construcción social del riesgo. Obtenido de Prevención de riesgos en el ecosistema por actividades de producción. https://bit.ly/3nF5xNr

Orozco, R., Meleán, R., y Rodríguez, G. (2004). Costos de producción en la cría de pollos de engorde. Revista Venezolana de Gerencia, $9(28), \quad 28$. https://doi.org/10.37960/revista.v9i28.9800

Perozo, E., y Nava, Á. (2005). El impacto de la gestión tecnológica en el contexto empresarial. $\begin{array}{lllll}\text { Revista Venezolana de Ciencias } & \text { Sociales, } & 9(2), & 18 .\end{array}$ https://www.redalyc.org/pdf/309/30990214.pdf 
Pesantez, J.-P., Ríos-Villacota, A., y González-Redrován, J. (2021). Integración de Sistemas Solares Fotovoltaicos en el Sector Camaronero Intensivo y Extensivo del Ecuador: Caso de Estudio en la Provincia de El Oro. Revista Politécnica, 47(2), 10. http://doi.org/10.33333/rp.vol47n2

Rincón-Soto, S.-M. A., Ximena, y LCardona-Restrepo, e. M. (2019). Clasificación teórica de los costos. Revista EAN, 14. https://doi.org/10.21158/01208160.n87.2019.2448

Ríos, G., y Gómez, L. (2008). Análisis de Costeo para un Sistema de Producción de Lechería Especializada “Un Acercamiento Al Análisis Económico En Ganadería De Leche”: Estudio De Caso. Dyna, 75(155), 10. https://www.redalyc.org/articulo.oa?id=49611953005

Rodríguez, G., Chiriboga, F., y Lojan, A. (2016). Las Camaroneras Ecuatorianas: Una Polémica Medioambiental. Revista Universidad y Sociedad, $8(3), \quad 6$. http://scielo.sld.cu/scielo.php?script=sci_arttext\&pid=S2218-36202016000300020

Rodríguez, L., Curetti, G., Garegnani, G., Grilli, G., Pastorella, F., y Paletto, A. (2016). La valoración de los servicios ecosistémicos en los ecosistemas forestales: un caso de estudio en Los Alpes Italianos. Bosque Valdivia), 37(1), 12. http://doi.org/10.4067/S071792002016000100005

Romero, N. (2014). Neoliberalismo e industria camaronera en Ecuador. Letras Verdes. Revista $\begin{array}{llll}\text { Latinoamericana de Estudios } & \text { Socioambientales, } & 24 .\end{array}$ https://revistas.flacsoandes.edu.ec/letrasverdes/article/view/1257/1137

Sorroza, L., Socola, M., Solano, G., y Echeverría, E. (2018). Evaluación del crecimiento y supervivencia de post-larvas en raceway. Cumbres, 5(1), 12. https://doi.org/10.48190/cumbres.v5n1a8

Tancara, C. (1993). La investigación Documental. Temas Sociales(17), 16. http://www.scielo.org.bo/scielo.php?script=sci_arttext\&pid=S0040-29151993000100008

Triviño, H., y Zhinin, E. (13 de Octubre de 2021). "Diseño de Prototipo de Un Sistema de Control de Alimentación y Monitoreo de Temperatura En el Proceso de Crianza de Larvas de Camarón en Estanques Empleando Tecnología Gsm-Gprs”. https://bit.ly/3nBMJib

UNIDO. (15 de agosto de 2020). Guía de recursos eficientes y producción más limpia sector camaronero. Obtenido de producción de camarón: https://bit.ly/3jv6CpA

Uzcátegui, C., Solano, J., y Figueroa, P. (2016). Perspectiva Sobre la Sostenibilidad de los Recursos Naturales a Largo Plazo Caso Industria Camaronera Ecuatoriana. Universidad y Sociedad, 8(3), $\quad$ 6. http://scielo.sld.cu/scielo.php?script=sci_abstract\&pid=S221836202016000300022

Varoni, G. A., y Rodríguez, C. A. (2017). Estrategias de crecimiento implementadas por los grupos económicos del Ecuador (2007-2016). Innovar, 27(65), 17. https://doi.org/10.15446/innovar.v27n65.65060

Villamar, C. (2004). Programa de bioseguridad para la cría de camarón orgánico Litopenaeus vannamei en cautiverio. AquaTIC, 11. 